\title{
EVALUACIÓN Y COMPARACIÓN DEL PROCESO DE REGENERACIÓN ÓSEA ENTRE INJERTO ÓSEO Y REPOSICIONAMIENTO DE HUESO CORTICAL
}

Gerardo Martínez-de la Cruz¹, Kensuke Yamauchi', Shinnosuke Nogami1, Yoshinaka Shimizu², Tetsu Takahashi1. División de Cirugía Oral y Maxilofacial, Departamento de Medicina Oral y Cirugía, Facultad de Odontología, Universidad de Tohoku, Sendai, Japón. División de Patología Oral, Departamento de Medicina Oral y Cirugía, Facultad de Odontología de la Universidad de Tohoku, Sendai, Japón.

\section{Introducción}

Los defectos horizontales y verticales de la cresta alveolar siguen siendo $\mathrm{u}$ desafió para la correcta colocación de implantes. Recientemente, la osteogénesi por medio de distracción del periostio para el aumento óseo ha sido sugerida como una nueva técnica. Hemos estado investigando métodos dinámicos y estáticos para la activación osteogénica del periostio. El reposicionamiento del hueso cortical (RHC) es una nueva técnica estática para regeneración ósea que combina aspectos de la distracción osteogénica (DO) e injerto óseo (IO) convencional, crea u espacio debajo del periostio y lo tensiona por medio del transporte y fijación de un segmento cortical.

\section{Objetivo}

Evaluar la capacidad para formar hueso por medio de RHC, como un nuevc método estático de regeneración ósea, y compararlo con el injerto ósec convencional.

\section{Metodología}

Experimento animal: 12 conejos blancos Japoneses (peso: $3 \mathrm{~kg}$ ) fueron dividido en 2 grupos; grupo experimental $(\mathrm{N}=9) \quad$ y grupo de control $(\mathrm{N}=3)$ Cada grupo contenía especímenes de 2,5 y 8 semanas.

\section{Diseño del estudio}

Osteotomía de la cortical ósea

La mitad de la cortical se posicióno como

IO sobre la superficie ósea y la otra mitad quedo elevada sobre el defecto óseo.

Se fijo con 2 tornillos de titanio.

En el grupo de control, se realizo osteotomía de la cortical ósea solamente, mas no se uso.

- 3 áreas de evaluación: IO, RCH, Defecto (D)

\section{Protocolo}
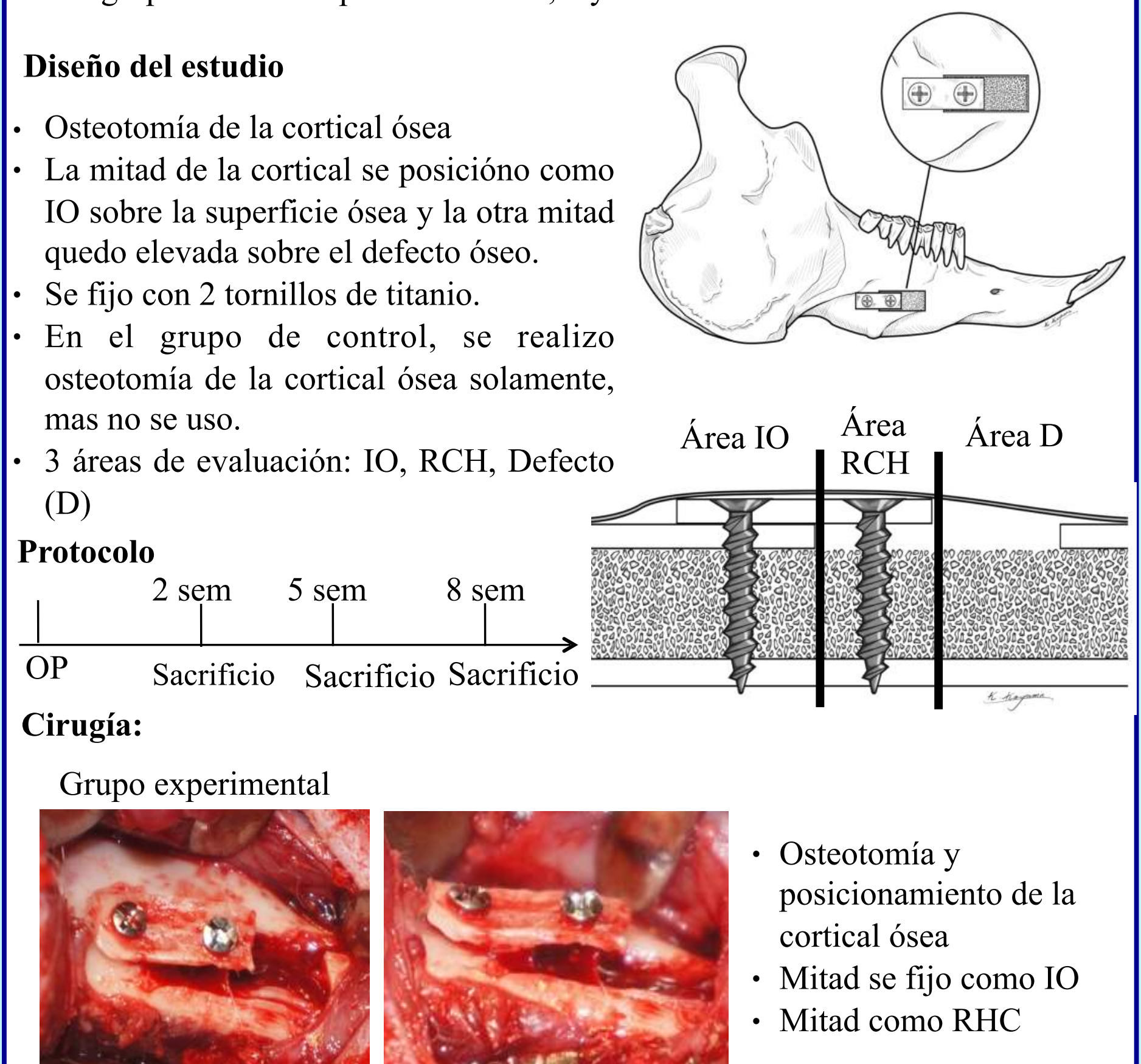

Gru
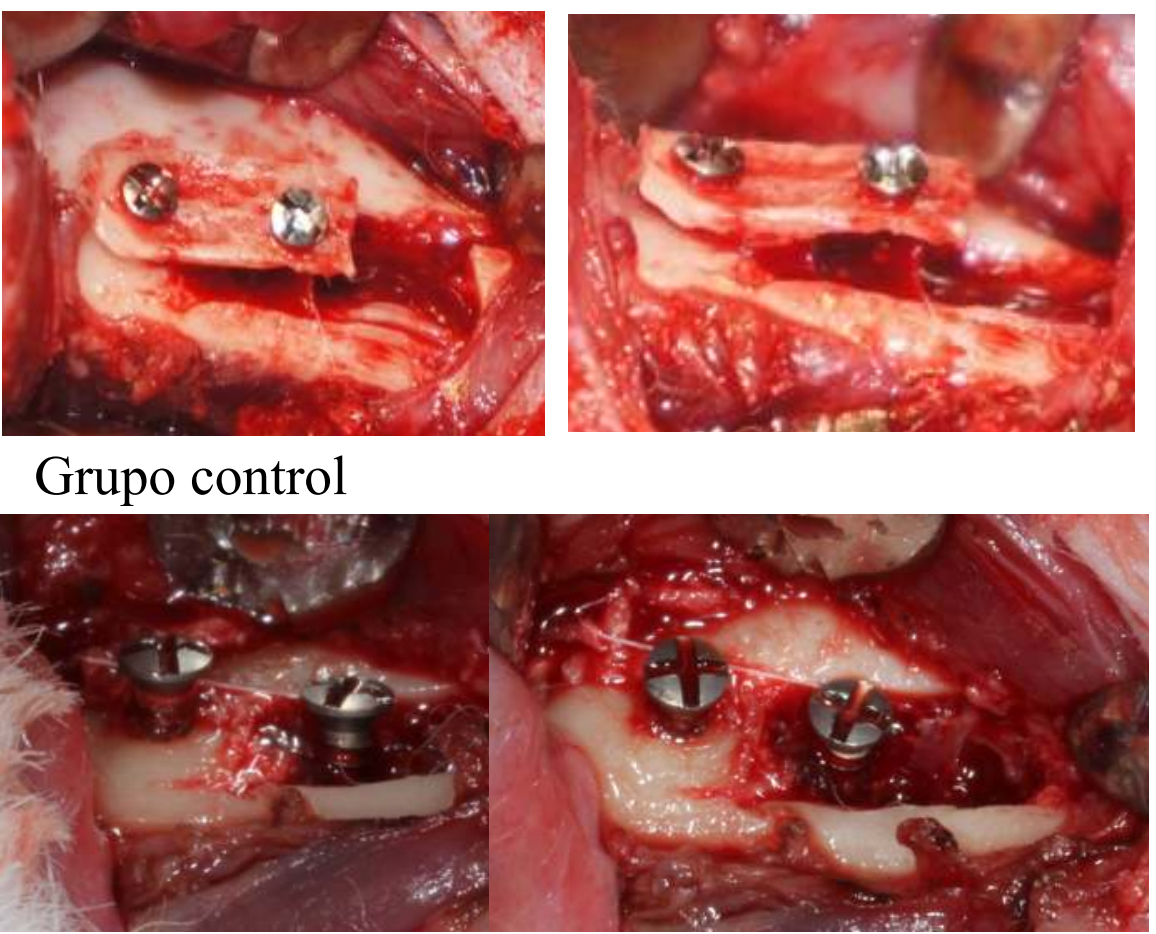

Osteotomía y

posicionamiento de la cortical ósea

- Mitad se fijo como IO

- Mitad como RHC

\section{Evaluación}
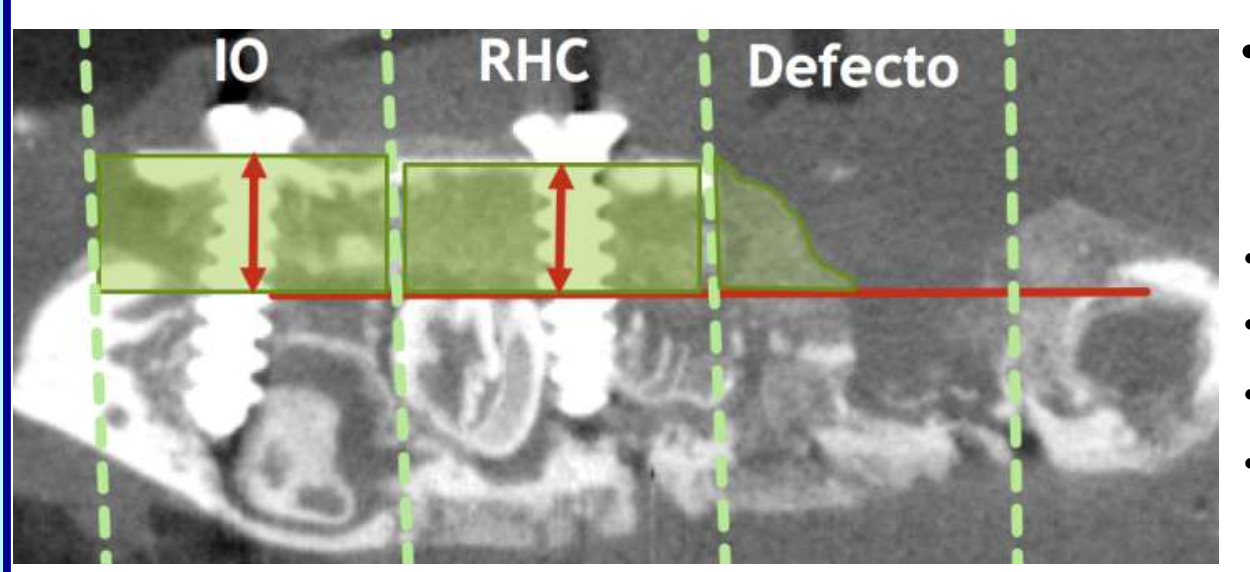

- La osteotomía también

fue hecha

No se uso la cortical ósea
(Comscantechno, Co, Japon) *ImageJ v1.44

Histomorfometrico

Tinciones H\&E y TRAP

Área $\left(\mathrm{mm}^{2}\right)$ y distanci (mm)

\section{Resultados}

\section{Resultados clínicos}

Grupo control
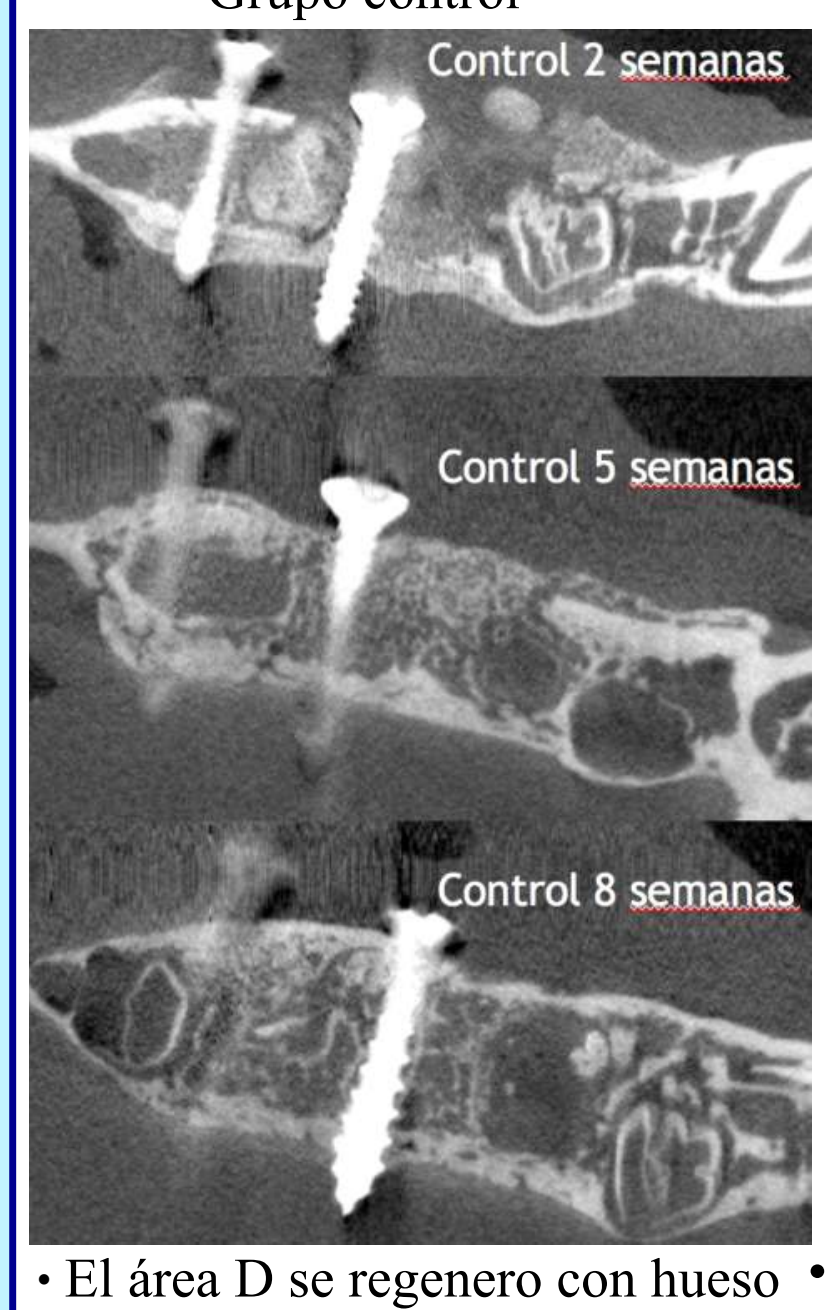

$$
\text { Hallazgos histologicos }
$$

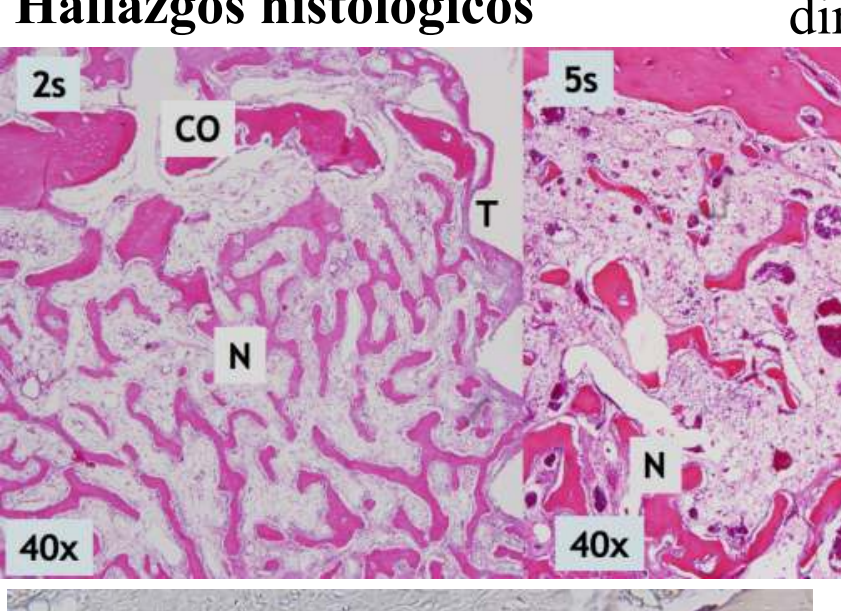

- 8ta semana, cabeza del tornillo en contacto directo con la superficie, poca reabsorción ósea
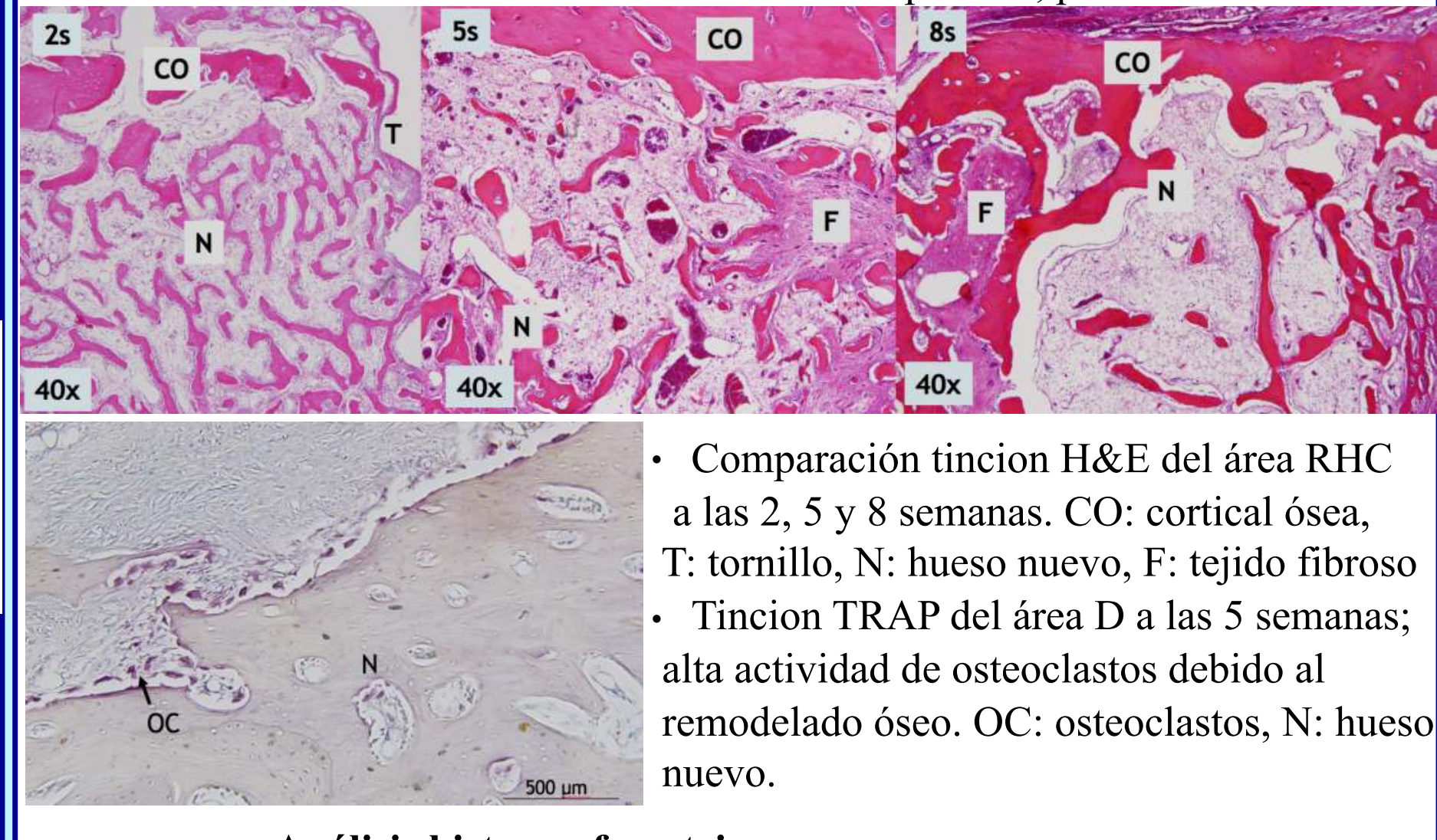

- Comparación tincion H\&E del área RHC a las 2, 5 y 8 semanas. CO: cortical ósea, $\mathrm{T}$ : tornillo, N: hueso nuevo, F: tejido fibroso - Tincion TRAP del área D a las 5 semanas; alta actividad de osteoclastos debido al remodelado óseo. OC: osteoclastos, N: hueso nuevo.

\begin{tabular}{|c|c|c|c|} 
Area $\mathrm{mm}^{2}$ & \multicolumn{3}{|c}{$\begin{array}{c}\text { Análisis histomorfometrico } \\
\text { Control }\end{array}$} \\
\hline & 10 & RHC & DEFECTO \\
\hline $2 \mathrm{~s}$ & $6.2 \pm 0.3$ & NA & NA \\
\hline $5 \mathrm{~s}$ & $6.3 \pm 0.5$ & $5.5 \pm 0.6$ & $5.7 \pm 0.4$ \\
\hline $\mathrm{s}$ & $4.7 \pm 0.2$ & $4.5 \pm 0.3$ & $6.2 \pm 0.5$ \\
\hline
\end{tabular}

Experimental

\begin{tabular}{|c|c|c|c|}
\hline & 10 & RHC & DEFECTO \\
\hline $2 \mathrm{~s}$ & $11.26 \pm 0.3$ & $12.7 \pm 2.6$ & $1.7 \pm 2.7$ \\
\hline $5 \mathrm{~s}$ & $13.23 \pm 5.2$ & $13.93 \pm 1.7 *$ & $8.1 \pm 4.5$ \\
\hline $8 \mathrm{~s}$ & $14.7 \pm 6.1 *$ & $13.16 \pm 1.9 *$ & $11.04 \pm 5.2$ \\
\hline
\end{tabular}

Tabla mostrando los resultados por área $(\mathrm{mm} 2)$, grupo y semana. - Prueba t de Student se utilizo para el análisis estadístico

- Existe una diferencia significativa en la cantidad de nuevo hueso respecto al grupo control

\section{Conclusión}

RHC es una técnica estática que utiliza 1 sola tracción para asegurar el espacio y tensionar el periostio, el cual se conoce tiene capacidad osteogénica al diferenciar células mesenquimales en osteoblastos y osteoclastos. En nuestro estudio, el área $\mathrm{D}$ demostró esta capacidad osteogénica del periostio al ser el área que formo mas hueso de la 2 a la 8 semana. Ventajas de RHC: no requiere donante, no necesita activación manual, solo 1 cirugia, utiliza mínimos materiales. Se necesitan mas estudios para determinar cuales son los limites y criterios de RHC para la activación osteogénica del periostio. 